\title{
Theoretical gravity and limb-darkening coefficients for the MOST satellite photometric system ${ }^{\star}$
}

\author{
A. Claret ${ }^{1}$, D. Dragomir ${ }^{2,3}$, and J. M. Matthews ${ }^{4}$ \\ ${ }^{1}$ Instituto de Astrofísica de Andalucía, CSIC, Apartado 3004, 18080 Granada, Spain \\ e-mail: claret@iaa.es \\ ${ }^{2}$ Las Cumbres Observatory Global Telescope, 6740 Cortona Drive, suite 102, Goleta CA 93117, USA \\ 3 Department of Physics, Broida Hall, UC Santa Barbara CA, USA \\ ${ }^{4}$ Department of Physics and Astronomy, University of British Columbia, Vancouver BC V6T1Z1, Canada
}

Received 27 January 2014 / Accepted 14 May 2014

\section{ABSTRACT}

\begin{abstract}
Aims. We present new calculations of limb and gravity-darkening coefficients to be used as input in many fields of stellar physics such as synthetic light curves of double-lined eclipsing binaries and planetary transits, studies of stellar diameters or line profiles in rotating stars.

Methods. We compute the limb-darkening coefficients specifically for the photometric system of the satellite MOST (Microvariability and Oscillations in STars). All computations were performed by adopting the least-square method, but for completeness we also performed calculations for the linear and bi-parametric approaches by adopting the flux conservation method. The passband gravitydarkening coefficients $y(\lambda)$ were computed by adopting a more general differential equation, which also takes the effects of convection into account.

Results. We used two stellar atmosphere models: ATLAS (plane-parallel) and PHOENIX (spherical and quasi-spherical). We adopted six laws to describe the specific intensity distribution: linear, quadratic, square root, logarithmic, exponential, and a more general one with four terms. The covered ranges of $T_{\mathrm{eff}}, \log g$, metallicities, and microturbulent velocities are $(1500-50000 \mathrm{~K}, 0-5.5,-5.0-+1.0$, $0-8 \mathrm{~km} \mathrm{~s}^{-1}$ ), respectively.
\end{abstract}

Key words. binaries: eclipsing - planetary systems - binaries: close - stars: atmospheres - stars: evolution

\section{Introduction}

As it is known, the limb-darkening coefficients (LDC) are important to investigate double-lined eclipsing binary light curves, stellar diameters, and line profiles in rotating stars. Other applications are related to gravitational micro-lensing or with optical interferometry. A more recent application comes from the analysis of extra-solar planets transits. Only a few years ago most of the light curves of double-lined eclipsing binaries, for example, did not have enough photometric quality to allow observational derivation of LDC and therefore, the semi-empirical data for limb-darkening were too scarce to perform a detailed comparison with the theoretical predictions. Fortunately, the situation is changing. Gradually, some high quality data are allowing these comparisons: for instance, light curves from double-lined eclipsing binaries (Claret 2008), planetary transits (Southworth 2008; Claret 2009; Sing 2010; Howarth 2011; Claret \& Bloemen 2011; Southworth 2012; Csizmadia et al. 2013; Müller et al. 2013), or microlensing events (Zub et al. 2011).

In this paper we continue our recent investigations on stellar atmospheres (Claret \& Bloemen 2011; Claret et al. 2012, 2013) extending the calculations of limb and gravity-darkening coefficients (GDC) to the photometric system of the satellite

* Tables 2-23 are only available at the CDS via anonymous ftp to cdsarc.u-strasbg.fr (130.79.128.5) or via http://cdsarc.u-strasbg.fr/viz-bin/qcat?J/A+A/567/A3
MOST (Microvariability and Oscillations in STars). The present calculations cover a wide range of values of $\log g, T_{\text {eff }}$, metallicities, and microturbulent velocities. We have used ATLAS (planeparallel) and PHOENIX (spherical and quasi-spherical) models. A description of both stellar atmosphere models can be found in the aforementioned papers and will not be repeated here. In the next sections, we introduce the numerical methods used to compute the LDC and GDC and discuss the results and the table organization.

\section{Numerical methods}

Here we adopt the classical approaches to the specific intensity distribution: the linear law

$\frac{I(\mu)}{I(1)}=1-u(1-\mu)$

the quadratic law

$\frac{I(\mu)}{I(1)}=1-a(1-\mu)-b(1-\mu)^{2}$,

the square root law

$\frac{I(\mu)}{I(1)}=1-c(1-\mu)-d(1-\sqrt{\mu})$, 


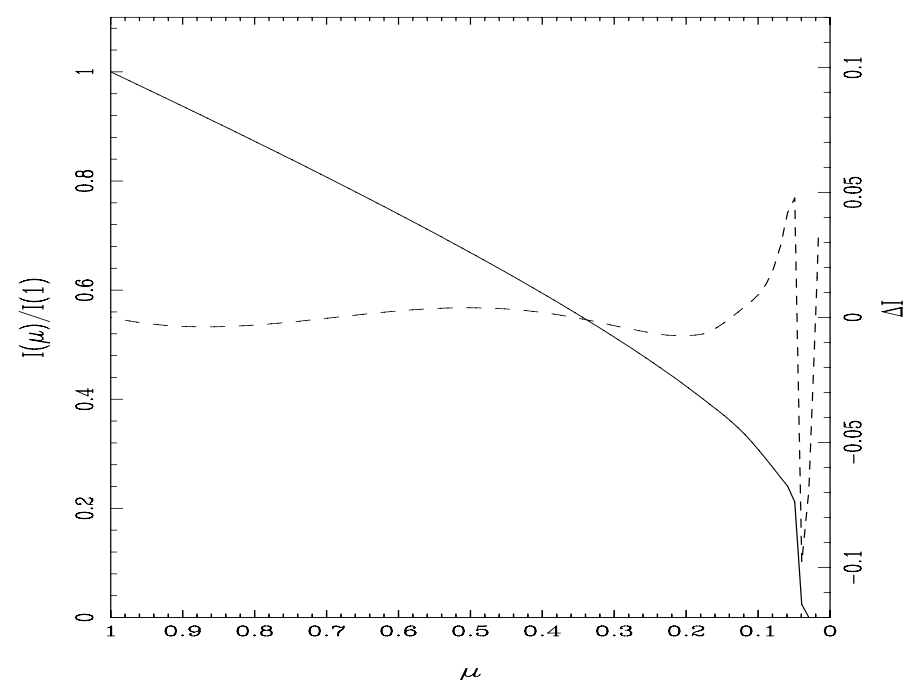

Fig. 1. Specific intensity distribution for a [4500 K, 4.5] PHOENIX spherical symmetric model. Continuous line represents the model intensities (left axis) while the dashed line denotes the deviations [model-fit] by adopting Eq. (6) (right axis). $\log [\mathrm{A} / \mathrm{H}]=0.0, V_{\xi}=2 \mathrm{~km} \mathrm{~s}^{-1}$. MOST photometric system, LSM.

the logarithmic law

$\frac{I(\mu)}{I(1)}=1-e(1-\mu)-f \mu \ln (\mu)$

the exponential law

$\frac{I(\mu)}{I(1)}=1-g(1-\mu)-\frac{h}{\left(1-e^{\mu}\right)}$

and a more general law with four terms

$\frac{I(\mu)}{I(1)}=1-\sum_{k=1}^{4} a_{k}\left(1-\mu^{\frac{k}{2}}\right)$

where $I(1)$ is the specific intensity at the centre of the disc, $u, a, b, c, d, e, f, g, h$, and $a_{k}$ are the corresponding LDC. The parameter $\mu$ is given by $\cos (\gamma)$, where $\gamma$ is the angle between the line of sight and the outward surface normal. All model atmosphere intensities were convolved with the transmission curve for MOST (Walker et al. 2003, Fig. 4 and Sect. 3.3). The calculations were performed by adopting the least-square method (LSM). The coefficients were also computed by using the flux conservation method (FCM) for bi-parametric and linear approximations, however, the fits using FCM provide larger scatter than the corresponding LSM, as previously discussed in the early papers of this series. We note that the PHOENIX models are separated in two parts: one with $1500 \mathrm{~K} \leq T_{\text {eff }} \leq 4800 \mathrm{~K}$ and another one with $5000 \mathrm{~K} \leq T_{\text {eff }} \leq 10000 \mathrm{~K}$. This is because these grids were computed with different $\mu$ and wavelength resolutions.

At this point, it is interesting and important to discuss some observational difficulties and their connections with the theoretical predictions. The observations of extrasolar planet transits and even of double-lined eclipsing are not accurate enough nowadays to detect the drop-off in the intensity predicted by spherical models near the limb (Fig. 1). Often, these observations are unevenly sampled, being more frequent near the centre of the stellar disc. In addition, it is not yet possible to infer from these observations more than bi-parametric semi-empirical LDC. On the

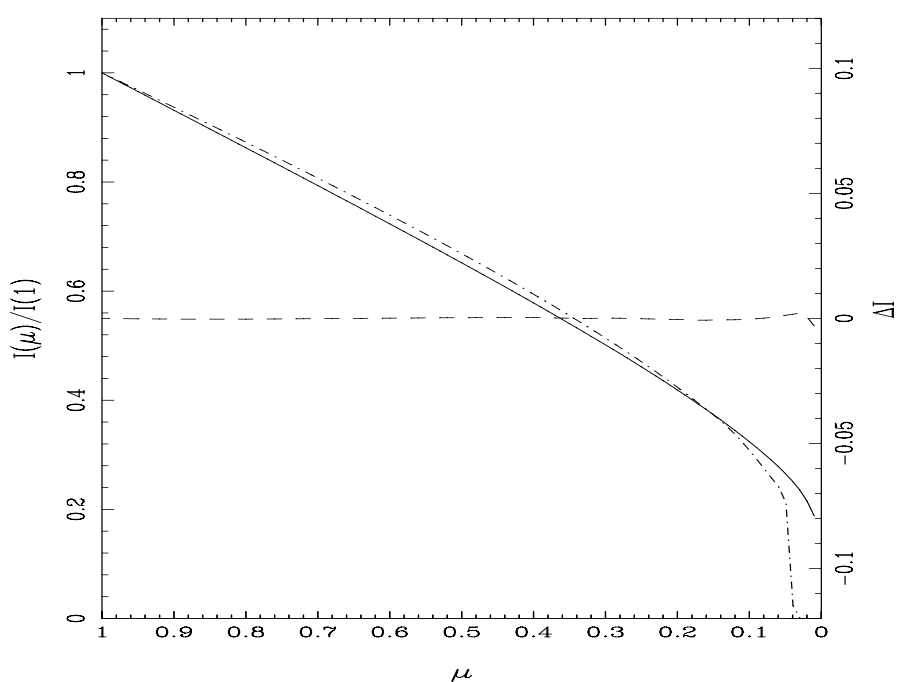

Fig. 2. Same as in Fig. 1 but for a plan-parallel ATLAS model (continuous line). The corresponding PHOENIX spherical symmetric model is also plotted to illustrate the similarities and differences in the intensity distribution (dash-dotted line). MOST photometric system, LSM.

other hand, the intensity distribution for spherical models cannot be well described by two terms laws, as we have shown in previous papers. As a consequence, a direct comparison between semi-empirical and theoretical spherical LDC, including the drop-off region, is not yet possible, however, even with the mentioned limitations one can test the spherical models against observations if we consider only the core of these models. The spherical models are constituted by two parts: the core $(\mu>0.1)$, which behaves like a plane-parallel structure, and the envelope, which delivers the spherical part of the intensities $(\mu \lesssim 0.1)$. The concept of quasi-spherical model (see below for a more detailed definition) may be useful to perform comparisons with semi-empirical LDC since bi-parametric laws can be applied to this kind of models with very good results (see Fig. 2).

Concerning the plan-parallel models such as ATLAS the situation is reversed with respect to the observational sampling: the $\mu$ points are more concentrated near the limb. Therefore, the LSM is not straightforward for these models. This concentration of points near the limb leads to weights that are too large for the mentioned region and consequently, to different LDC. In order to prevent weights that are too large to the limb in the calculations of our theoretical LDC using LSM we adopted equally spaced $\mu$ points. However, even when one adopts a given law and atmosphere model, different numerical methods and/or $\mu$ distribution may produce different LDC. On the other hand, as already mentioned, the observations often are not evenly sampled. Thus, the comparison theory-observation of LDC is not a simple and direct task. Howarth (2011), in a paper on exoplanetary transits, introduced the synthetic-photometry/atmosphere-model (SPAM) method that combines semi-empirical information and theoretical LDC. Following this author the resulting LDC can reasonably be compared directly with empirical values. We think that these issues could be solved (at least partially) through a closer interaction between the theoretical and observational astrophysicists since to be fully consistent with uneven observational data, it would be necessary to compute the theoretical LDC, under different assumptions, for each set of observations. Part of the ingredients to carry out such a comparison are available in this series of papers on limb and gravity-darkening inasmuch as we provide bi-parametric and linear LDC computed 
through two different numerical methods, for example, leastsquare and flux conservation. We are open for collaboration to try to improve the levels of confidence in this comparison.

\section{The MOST satellite and the need for customized limb-darkening coefficients}

The MOST satellite (Matthews et al. 2004; Walker et al. 2003) is in a Sun-synchronous polar orbit around the Earth with a period of $101.4 \mathrm{~min}$, and carries a Maksutov-Cassegrain $15 \mathrm{~cm}$ telescope. The satellite's orbit results in a continuous viewing zone (CVZ) ranging in declination from -19 to $36^{\circ}$. It can observe stars within the CVZ continuously for up to 8 weeks. The telescope is equipped with a broad-band filter spanning the wavelength range between $350 \mathrm{~nm}$ and $750 \mathrm{~nm}$ and can observe either in the direct imaging or Fabry imaging mode. The Fabry imaging mode, in which an image of the telescope pupil is focused on the CCD by each of the Fabry microlenses in a $6 \times 6$ array, is employed for stars brighter than $V \approx 6$.

In addition to its stellar variability and asteroseismology observing programs, MOST has made several significant contributions to the study of eclipsing binaries and especially transiting extrasolar planets since its launch in 2003. Kaminski et al. (2007) have used MOST photometry to characterize stellar activity in the V471 Tau white dwarf-red dwarf binary. Rowe et al. (2008) constrained the albedo of the much-studied exoplanet HD 209458b to less than 0.1, thus providing direct observational evidence that hot gaseous exoplanets may be relatively dark. In 2011, MOST monitored the 55 Cancri system, a campaign that led to the discovery of the first transiting superEarth orbiting a very bright star (Winn et al. 2011). Two years later, the telescope was used to discover the transiting nature of HD 97658b, which thus became the second super-Earth known to transit a very bright star (Dragomir et al. 2013).

These works are only a selection of the MOST publications that have either made use of or would have benefited from using theoretical LDC. Often these analyses have employed linear LDC roughly approximated for the MOST bandpass (Kaminski et al. 2007), or resorted to constraining the LDC using the data itself. Neither method is ideal, particularly the latter, which has been used with low signal-to-noise data.

The MOST observations of exoplanet transits often lack the precision required to significantly constrain the limb darkening of the host star. This is especially true for shallow transits like those of the super-Earths $55 \mathrm{Cnc}$ e and HD 97658b. For these planets, using theoretical LDC provides a significant improvement in the transit parameters over fitting them as free parameters. Csizmadia et al. (2013) find that to constrain LDC to better than the current uncertainties on their theoretical values, a signal-to-noise ratio $(\mathrm{S} / \mathrm{N})$ higher than about 20 is required. The MOST discovery photometry of super-Earth transits usually has a SNR lower than 20 and we find it is therefore more appropriate to use theoretical LDC values. In addition to precision, the accuracy of the LDC is also a matter of concern. Müller et al. (2013) have looked at this issue, and recommend fixing the LDC to their theoretical values for data with $\mathrm{S} / \mathrm{N}$ lower than that of the Kepler data used in their study. This is the case for MOST transit photometry for which we also find that the uncertainties in the transit parameters are of the order of a few percent, significantly larger than the $\sim 1 \%$ error they find is introduced in the transit parameters by the differences between measured and theoretical LDC. In any case, it is always good practice to compare results derived both from fitting and fixing the LDC, thus the need for theoretical values.
In the foreseeable future we anticipate more projects that will require the use of theoretical LDC values for MOST data, including upcoming analyses of new and existing photometry of 55 Cnc e and HD 97658b, and the discovery of any new transiting planets as part of an ongoing transit search.

\section{Discussion of the results: limb-darkening}

As an example of the present calculations, we show in Fig. 1 the intensity distribution for a spherically symmetrical PHOENIX model with $T_{\text {eff }}=4500 \mathrm{~K}$ and $\log g=4.5$. The model intensity is represented by a continuous line while the dashed one denotes the deviation of the intensity [model-fitted] by adopting Eq. (6). The largest deviations come from the region near the limb, i.e., for $\mu<0.1$. In spite of the steeper profile at the dropoff zone, the agreement can be considered as good, as previously shown for other photometric systems (Claret et al. 2012, 2013). The validity of Eq. (6) was confirmed in other papers on limbdarkening and very recently by Magic et al. (2014) for 3D model atmospheres.

The behaviour of a similar model but computed with the ATLAS code is shown in Fig. 2 where the equivalent PHOENIX model is also shown to illustrate their similarities and differences. We note that Eq. (6) provides an almost perfect fit to the actual intensities for the plan-parallel model. Such a very good fit is due to the simpler intensity distribution of plane-parallel model if compared with the equivalent spherical model. By inspecting Fig. 2, we can see that both models present a very similar intensity distribution (mainly the slope) for $\mu>0.1$. Although both models were computed with different $\mu$ resolution, different set of opacities, $\lambda$ resolution, mixinglength parameters and, mainly with different geometries, the similarity is remarkable. This similarity allowed us to define a quasi-spherical model (Claret \& Hauschildt 2003) as one computed using spherical symmetry but whose LDC are computed without considering the points inside the drop-off region. Such a concept is useful, for example, where the effects of sphericity are not important. We apply this concept when we compare the LDC for PHOENIX and ATLAS models in the next paragraphs.

As known, the linear law is not a good approximation for most of the intensity distributions, however, because of its simplicity it is useful to compare LDC generated with different stellar atmosphere codes. In Fig. 3 we show the linear LDC for quasi-spherical PHOENIX models and ATLAS models with $\log g=4.5$, typically for main-sequence stars. Although PHOENIX and ATLAS models are computed with different input physics, Fig. 3 illustrates that both atmospheres models give similar results (see also Fig. 2 in Claret et al. 2013, for other photometric systems). The largest deviation near $\log T_{\text {eff }} \approx 3.9$ is due to the onset of convection since the models were computed with different mixing-length parameter (2.00 and 1.25 , for PHOENIX and ATLAS, respectively. The mentioned similarities in the corresponding LDC also serve as additional supports to the concept of quasi-spherical models.

The effective wavelengths for MOST and Kepler are somewhat different $(\approx 560$ and $630 \mathrm{~nm})$ but it is interesting to compare the corresponding theoretical LDC. This is done in Fig. 4 where the linear LDC for the MOST models are indicated by a continuous line while the Kepler ones are represented by a dashed line. There is a clear difference, the Kepler $u$ being systematically smaller than MOST linear LDC. The difference is $\approx 0.05$ for hotter models but it increases up to 0.10 or more for lower effective temperatures. In the later case the differences are of the order of the average semi-empirical error bar in the linear LDCs. 


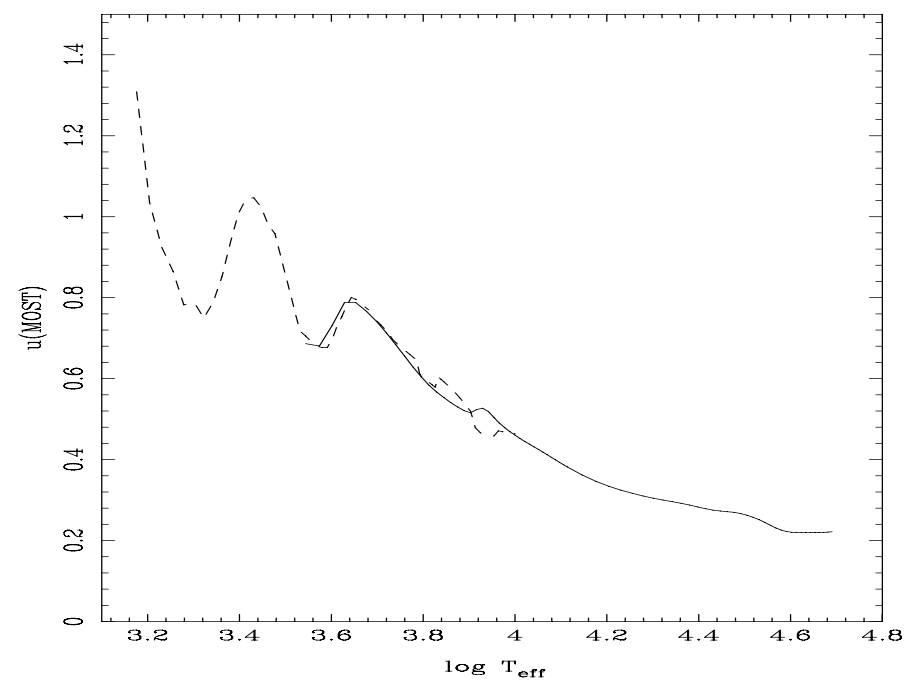

Fig. 3. Theoretical linear LDC for ATLAS models (continuous line) and PHOENIX quasi-spherical models (dashed line). Log $g=4.5$, $\log [\mathrm{A} / \mathrm{H}]=0.0, V_{\xi}=2 \mathrm{~km} \mathrm{~s}^{-1}$. MOST photometric system, LSM.

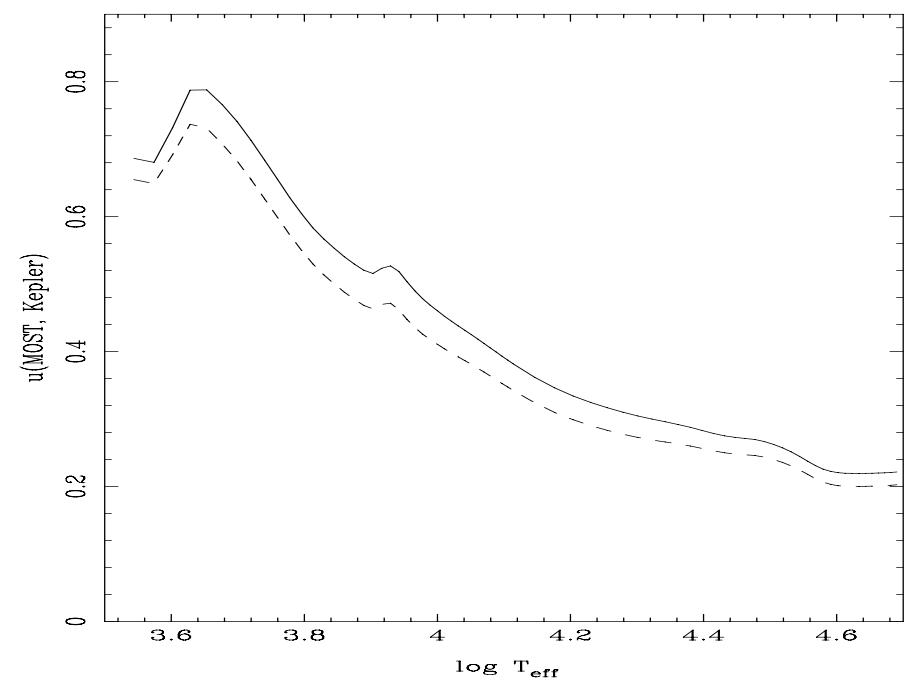

Fig. 4. Theoretical linear LDC for ATLAS models. Continuous line indicated the results for MOST while dashed line represents Kepler calculations. $\log g=4.5, \log [\mathrm{A} / \mathrm{H}]=0.0, V_{\xi}=2 \mathrm{~km} \mathrm{~s}^{-1}$, LSM.

We note that the differences between the linear LDC for MOST and Kepler for ATLAS models are always larger than those between ATLAS and PHOENIX, at least for the overlapping region studied here. It would be very interesting and useful if MOST and Kepler users contrasted their semi-empirical LDC for similar host stars.

As mentioned in the Introduction, the information on semiempirical LDC is increasing very rapidly. One of the most relevant papers on this subject is by Southworth (2012) who analysed 38 transits of exoplanets, paying special attention to limb-darkening. More recently, Müller et al. (2013) also investigated transits of 38 Kepler planetary candidates. On the other hand, Csizmadia et al. (2013) studied the effects of the LDC on the accuracy of exoplanets radii. While Müller and collaborators advocate the adoption of the theoretical LDC as fixed parameters mainly for those systems with high impact parameter, Csizmadia et al. recommend the adjustment of these coefficients. It is beyond of scope of this paper to discuss this controversy but

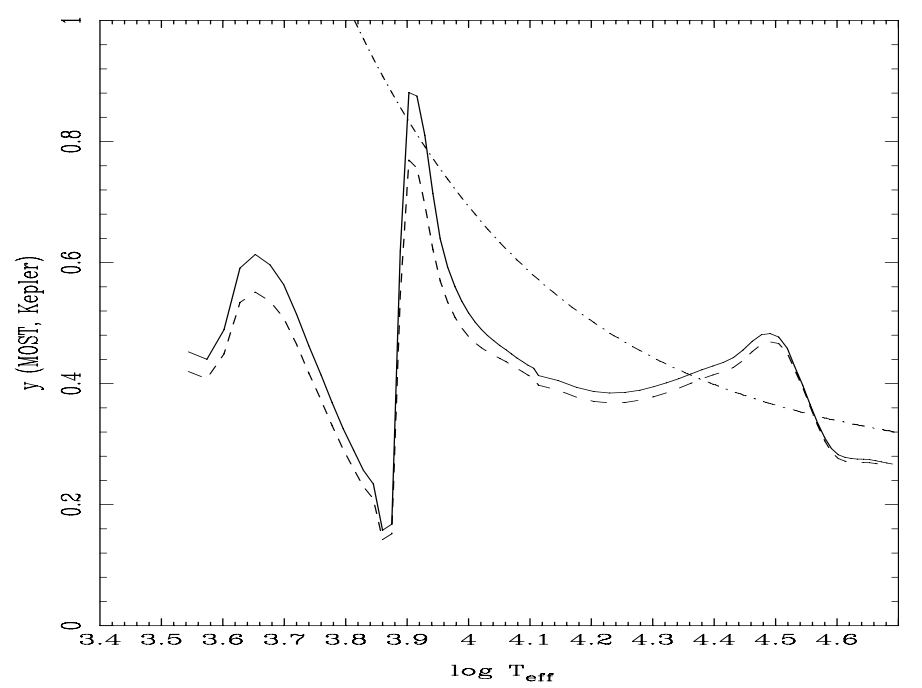

Fig. 5. Theoretical gravity-darkening coefficients for ATLAS models. The continuous line represents the calculations for MOST and dashed line denotes Kepler results. The dash-dotted line indicates the GDC for the black-body approach at $\lambda=563 \mathrm{~nm}$. $\log g=4.5, \log [\mathrm{A} / \mathrm{H}]=0.0$, $V_{\xi}=2 \mathrm{~km} \mathrm{~s}^{-1}$, LSM.

in our opinion, both methods are not exclusive. The current theoretical LDC should be used together with the free parameters procedure. Comparing the results provided by both methods one could try to quantify the level of discrepancies that may be useful to provide clues to improve the atmosphere models, numerical methods, or even the observational methodology.

\section{Discussion of the results: gravity-darkening coefficients}

The GDC are an important tool to compute the light distribution in distorted stellar configurations (both tidal and rotational). Originally, they were computed by assuming black-body radiation (Kopal 1959), however, as shown in Claret (2003), this approach is not a good one and the introduction of more elaborated stellar atmosphere models was needed. To provide MOST users of more realistic calculations we have also computed the passband GDC $y(\lambda)$ using the complete grid of ATLAS models. We adopt the following differential equation, which was introduced systematically in GDC calculations by Claret \& Bloemen (2011)

$y\left(\lambda, T_{\text {eff }}, Z, \log g, V_{\xi}\right)=\left(\frac{\partial \ln I(\lambda)}{\partial \ln g}\right)_{T_{\text {eff }}}+\left(\frac{\mathrm{d} \ln T_{\text {eff }}}{\mathrm{d} \ln g}\right)\left(\frac{\partial \ln I(\lambda)}{\partial \ln T_{\text {eff }}}\right)_{g}$,

where $\lambda$ denotes the passband, $Z$ the metal content, $V_{\xi}$ the microturbulent velocity, and $I$ the intensity at a given passband at the centre of the stellar disc. The effects of convection in Eq. (7), through the term $\left(\frac{\mathrm{d} \ln T_{\mathrm{eff}}}{\mathrm{d} \ln g}\right)$, are taken into account following Claret $\&$ Bloemen (2011), where more details on the calculation of $y(\lambda)$ can be found.

Figure 5 illustrates the behaviour of $y$ for MOST and Kepler photometric systems. The black-body approach is represented by a dashed-dotted line. Clearly, such an approximation is not good even for hotter models, specially for shorter wavelengths. Therefore, we recommend the adoption of calculations that consider modern stellar atmosphere models. On the other hand, the behaviour of $y$ is similar for MOST and Kepler except for a 
Table 1. Gravity and limb-darkening coefficients for the MOST photometric system.

\begin{tabular}{|c|c|c|c|c|c|c|c|}
\hline Name & Source & Range $T_{\text {eff }}$ & Range $\log g$ & $\log [\mathrm{M} / \mathrm{H}]$ & Vel Turb. & Filter & Fit/equation/model \\
\hline Table 2 & PHOENIX & $1500 \mathrm{~K}-4800 \mathrm{~K}$ & $2.5-5.5$ & 0.0 & $2 \mathrm{~km} \mathrm{~s}^{-1}$ & MOST & LSM/FCM/Eq. (1) quasi-spherical \\
\hline Table 3 & PHOENIX & $1500 \mathrm{~K}-4800 \mathrm{~K}$ & $2.5-5.5$ & 0.0 & $2 \mathrm{~km} \mathrm{~s}^{-1}$ & MOST & LSM/FCM/Eq. (2) quasi-spherical \\
\hline Table 4 & PHOENIX & $1500 \mathrm{~K}-4800 \mathrm{~K}$ & $2.5-5.5$ & 0.0 & $2 \mathrm{~km} \mathrm{~s}^{-1}$ & MOST & LSM/FCM/Eq. (3) quasi-spherical \\
\hline Table 5 & PHOENIX & $1500 \mathrm{~K}-4800 \mathrm{~K}$ & $2.5-5.5$ & 0.0 & $2 \mathrm{~km} \mathrm{~s}^{-1}$ & MOST & LSM/FCM/Eq. (4) quasi-spherical \\
\hline Table 6 & PHOENIX & $1500 \mathrm{~K}-4800 \mathrm{~K}$ & $2.5-5.5$ & 0.0 & $2 \mathrm{~km} \mathrm{~s}^{-1}$ & MOST & LSM/Eq. (5) quasi-spherical \\
\hline Table 7 & PHOENIX & $1500 \mathrm{~K}-4800 \mathrm{~K}$ & $2.5-5.5$ & 0.0 & $2 \mathrm{~km} \mathrm{~s}^{-1}$ & MOST & LSM/Eq. (6) quasi-spherical \\
\hline Table 8 & PHOENIX & $1500 \mathrm{~K}-4800 \mathrm{~K}$ & $2.5-5.5$ & 0.0 & $2 \mathrm{~km} \mathrm{~s}^{-1}$ & MOST & LSM/Eq. (5) spherical \\
\hline Table 9 & PHOENIX & $1500 \mathrm{~K}-4800 \mathrm{~K}$ & $2.5-5.5$ & 0.0 & $2 \mathrm{~km} \mathrm{~s}^{-1}$ & MOST & LSM/Eq. (6) spherical \\
\hline Table 10 & PHOENIX & $5000 \mathrm{~K}-10000 \mathrm{~K}$ & $3.0-5.5$ & 0.0 & $2 \mathrm{~km} \mathrm{~s}^{-1}$ & MOST & LSM/FCM/Eq. (1) quasi-spherical \\
\hline Table 11 & PHOENIX & $5000 \mathrm{~K}-10000 \mathrm{~K}$ & $3.0-5.5$ & 0.0 & $2 \mathrm{~km} \mathrm{~s}^{-1}$ & MOST & LSM/FCM/Eq. (2) quasi-spherical \\
\hline Table 12 & PHOENIX & $5000 \mathrm{~K}-10000 \mathrm{~K}$ & $3.0-5.5$ & 0.0 & $2 \mathrm{~km} \mathrm{~s}^{-1}$ & MOST & LSM/FCM/Eq. (3) quasi-spherical \\
\hline Table 13 & PHOENIX & $5000 \mathrm{~K}-10000 \mathrm{~K}$ & $3.0-5.5$ & 0.0 & $2 \mathrm{~km} \mathrm{~s}^{-1}$ & MOST & LSM/FCM/Eq. (4) quasi-spherical \\
\hline Table 14 & PHOENIX & $5000 \mathrm{~K}-10000 \mathrm{~K}$ & $3.0-5.5$ & 0.0 & $2 \mathrm{~km} \mathrm{~s}^{-1}$ & MOST & LSM/Eq. (5) quasi-spherical \\
\hline Table 15 & PHOENIX & $5000 \mathrm{~K}-10000 \mathrm{~K}$ & $3.0-5.5$ & 0.0 & $2 \mathrm{~km} \mathrm{~s}^{-1}$ & MOST & LSM/Eq. (6) quasi-spherical \\
\hline Table 16 & PHOENIX & $5000 \mathrm{~K}-10000 \mathrm{~K}$ & $3.0-5.5$ & 0.0 & $2 \mathrm{~km} \mathrm{~s}^{-1}$ & MOST & LSM/Eq. (5) spherical \\
\hline Table 17 & PHOENIX & $5000 \mathrm{~K}-10000 \mathrm{~K}$ & $3.0-5.5$ & 0.0 & $2 \mathrm{~km} \mathrm{~s}^{-1}$ & MOST & LSM/Eq. (6) spherical \\
\hline Table 18 & ATLAS & $3500 \mathrm{~K}-50000 \mathrm{~K}$ & $0.0-5.0$ & $-5.0-+1.0$ & $0-8 \mathrm{~km} \mathrm{~s}^{-1}$ & MOST & LSM/FCM/Eq. (1) \\
\hline Table 19 & ATLAS & $3500 \mathrm{~K}-50000 \mathrm{~K}$ & $0.0-5.0$ & $-5.0-+1.0$ & $0-8 \mathrm{~km} \mathrm{~s}^{-1}$ & MOST & LSM/FCM/Eq. (2) \\
\hline Table 20 & ATLAS & $3500 \mathrm{~K}-50000 \mathrm{~K}$ & $0.0-5.0$ & $-5.0-+1.0$ & $0-8 \mathrm{~km} \mathrm{~s}^{-1}$ & MOST & LSM/FCM/Eq. (3) \\
\hline Table 21 & ATLAS & $3500 \mathrm{~K}-50000 \mathrm{~K}$ & $0.0-5.0$ & $-5.0-+1.0$ & $0-8 \mathrm{~km} \mathrm{~s}^{-1}$ & MOST & LSM/FCM/Eq. (4) \\
\hline Table 22 & ATLAS & $3500 \mathrm{~K}-50000 \mathrm{~K}$ & $0.0-5.0$ & $-5.0-+1.0$ & $0-8 \mathrm{~km} \mathrm{~s}^{-1}$ & MOST & LSM/Eq. (6) \\
\hline Table 23 & ATLAS & $3500 \mathrm{~K}-50000 \mathrm{~K}$ & $0.0-5.0$ & $-5.0-+1.0$ & $0-8 \mathrm{~km} \mathrm{~s}^{-1}$ & MOST & Gravity-darkening coefficients $y$ \\
\hline
\end{tabular}

systematic difference which decreases with the effective temperature, as expected. The jump in $y$ around $\log T_{\text {eff }} \approx 3.9$ probably is connected with the onset of convection. This effect is also detected in the LDC calculations, as already noted by Díaz-Cordovés et al. (1995). Although not shown in the figure, the differences in $y$ computed using PHOENIX and ATLAS models are small for a given photometric system since the GDC calculations use only information from the centre of the disc. Equation (7) indicates that the GDC also depend on the metallicity, surface gravity, and microturbulent velocities. These dependencies are generally small, except for colder models.

\section{Summary and table organization}

The LDC for the spherical and quasi-spherical PHOENIX models as well as for the plan-parallel ATLAS grid are presented for the photometric system of the MOST space mission. Six equations were adopted to describe the intensity distribution of the mentioned models. All calculations were performed using the least-square method though, for completeness, we also present LCD based on bi-parametric and linear laws using the flux conservation method.

Computations of GDE are also presented for the same models (except PHOENIX). These calculations make use of a new differential equation that takes the effects of the term $\left(\frac{\partial \ln I(\lambda)}{\partial \ln g}\right)_{T_{\text {eff }}}$ and of convection through the quantity $\left(\frac{\mathrm{d} \ln T_{\text {eff }}}{\mathrm{d} \ln g}\right)$ into account.

Table 1 summarizes the data available at CDS or directly from the authors. Additional calculations of LDC and/or GDC can be provided to interested readers on request.
Acknowledgements. We acknowledge P. Hauschildt and S. Witte for providing the PHOENIX models as well as the anonymous referee for his(her) useful suggestions and comments. We also would like to thank B. Rufino for useful comments. The Spanish MEC (AYA2012-39727-C03-01) is gratefully acknowledged for its support during the development of this work. This research has made use of the SIMBAD database, operated at the CDS, Strasbourg, France, and of NASA's Astrophysics Data System Abstract Service.

\section{References}

Csizmadia, Sz., Pasternacki, Th., Dreyer, C., et al. 2013, A\&A, 549, A9 Claret, A. 2003, A\&A, 406, 623

Claret, A. 2008, A\&A, 482, 259

Claret, A. 2009, A\&A, 506, 1335

Claret, A., \& Bloemen, S. 2011, A\&A, 529, A75

Claret, A., \& Hauschildt, P. H. 2003, A\&A, 412, 241

Claret, A., Hauschildt, P. H., \& Witte, S. 2012, A\&A, 546, A14

Claret, A., Hauschildt, P. H., \& Witte, S. 2013, A\&A, 552, A16

Desmet, M., Aerts, C., Matthews, J. M., et al. 2009, AIP Conf. Proc., 1170, 376 Díaz-Cordovés, J., Claret, A., \& Giménez, A. 1995, A\&AS, 110, 329

Dragomir, D., Matthews, J. M., Eastman, J. D., et al. 2013, ApJ, 772, L2 Howarth, I. D. 2011, MNRAS, 418, 1165

Kaminski, K. Z., Rucinski, S. M., \& Matthews, J. M. 2007, AJ, 134, 1206

Kopal, Z. 1959, Close Binary Systems (Chapman \& Hall)

Magic, Z., Chiavassa, A., Collet, R., \& Asplund, M. 2014, A\&A, accepted [arXiv: 1403 . 3487]

Matthews, J. M., Kuschnig, R., Guenther, D. B., et al. 2004, Nature, 430, 921

Müller, H. M., Huber, K. F., Czesla, S., Wolter, U., \& Schmitt, J. H. M. M. 2013, A\&A, 560, A112

Rowe, J. F., Matthews, J. M., Seager, S., et al. 2008, ApJ, 689, 1345

Sing, D. K. 2010, A\&A, 510, A21

Southworth, J. 2008, MNRAS, 386, 1644

Southworth, J. 2012, MNRAS, 426, 1291

Walker, G., Matthews, J. M., Kuschnig, R., et al. 2003, PASP, 115, 1023

Winn, J. N., Matthews, J. M., Dawson, R. I., et al. 2011, ApJ, 737, L18

Zub, M., Cassan, A., Heyrovsky, D., et al. 2011, A\&A, 525, A15 